Originalveröffentlichung in: Mueller-Goldingen, Christian ; Stier, Kurt (Hrsgg.): Lēnaika :

Festschrift für Carl Werner Müller zum 65. Geburtstag am 28. Januar 1996, Stuttgart 1996,

S. 365-383 (Beiträge zur Altertumskunde ; 89)

\title{
ZEITGESTALT UND FARBE, PLASTIK UND HELLDUNKEL ALS DIMENSIONEN DER VERANSCHAULICHUNG MYTHISCHER UND CHRISTLICHER THEMEN IN DER MALEREI
}

\author{
von Lorenz Dittmann
}

Die neuere Erfahrung von Zeit, in deren Bahnen auch das 20. Jahrhundert noch steht, formulierte als erster Kant in seiner „Kritik der reinen Vernunft“ von 1781 und 1787. Hier heißt es in $\S 6$ : „Die Zeit ist nichts anders, als die Form des innern Sinnes, d.i. des Anschauens unserer selbst und unsers innern Zustandes. Denn die Zeit kann keine Bestimmung äußerer Erscheinungen sein; sie gehöret weder zu einer Gestalt, oder Lage etc., dagegen bestimmt sie das Verhältnis der Vorstellungen in unserm innern Zustande. Und, eben, weil diese innre Anschauung keine Gestalt gibt, suchen wir auch diesen Mangel durch Analogien zu ersetzen, und stellen die Zeitfolge durch eine ins Unendliche fortgehende Linie vor, in welcher das Mannigfaltige eine Reihe ausmacht, die nur von einer Dimension ist, und schließen aus den Eigenschaften dieser Linie auf alle Eigenschaften der Zeit, außer den einigen, daß die Teile der erstern zugleich, die der letztern aber jederzeit nach einander sind. Hieraus erhellt auch, daß die Vorstellung der Zeit selbst Anschauung sei, weil alle ihre Verhältnisse sich an einer äußern Anschauung ausdrücken lassen.

Die Zeit ist die formale Bedingung a priori aller Erscheinungen überhaupt. Der Raum, als die reine Form aller äußeren Anschauung ist als Bedingung a priori bloß auf äußere Erscheinungen eingeschränkt. Dagegen, weil alle Vorstellungen, sie mögen nun äußere Dinge zum Gegenstande haben, oder nicht, doch an sich selbst, als Bestimmungen des Gemüts, zum inneren Zustande gehören; dieser innere Zustand aber unter der formalen Bedingung der innern Anschauung, mithin der Zeit gehöret: so ist die Zeit eine Bedingung a priori von aller Erscheinung überhaupt, und zwar die unmittelbare Bedingung der inneren (unserer Seelen) und eben dadurch mittelbar auch der äußern Erscheinungen. Wenn ich a priori sagen kann: alle äußere Erscheinungen sind im Raume, und nach den Verhältnissen des Raumes a priori bestimmt, so kann ich aus dem Prinzip des innern Sinnes ganz allgemein sagen: alle Erscheinungen 
überhaupt, d.i. alle Gegenstände der Sinne, sind in der Zeit, und stehen notwendiger Weise in Verhältnissen der Zeit." ${ }^{\text {"1 }}$

Diese Sätze machen deutlich, weshalb auch die Interpretation von Werken der Malerei nicht auf eine Analyse ihrer Zeitgestalt verzichten kann. Malerei stellt mit den „äußeren Erscheinungen“ immer auch die menschliche Existenz mit dar. Die Wesensbestimmungen dieser Existenz, Freiheit und Tod, Schicksal, Ewigkeit, Wachstum, Erinnern, Vergessen, Hoffen usf. sind, als Zustände oder Ziele des „inneren Sinnes“, des „Gemütes“, unabtrennbar von der „Anschauungsform“ des „inneren Sinnes", der Zeit.

Das Konzept von Zeit als „innerem Sinn“ ist jedoch zu ergänzen durch Konzepte transsubjektiver Zeiten². Für das Verständnis von Werken christlicher Thematik ist es von großer Bedeutung, sich die Zeitauffassung des Neuen Testaments zum Bewußtsein zu bringen.

Wichtig innerhalb dieses Problembereichs ist das Buch von Oscar Cullmann: „Christus und die Zeit. Die urchristliche Zeit- und Geschichtsauffassung “3. „Das Besondere an der christlich-heilsgeschichtlichen Zeitauffassung ist“, so Cullmann, „doppelter Art“: „Erstens ist das Heil gebunden an ein fortlaufendes, Vergangenheit, Gegenwart und Zukunft umfassendes Zeitgeschehen, Offenbarung und Heil erfolgen auf einer ansteigenden Zeitlinie ... Der streng geradlinig lineare Zeitbegriff des Neuen Testaments“ grenzt sich damit ab ,gegenüber dem griechischen zyklischen und gegenüber aller Metaphysik, wo das Heil immer im ,Jenseits " verfügbar ist". „Offenbarung und Heil ,geschehen" nach urchristlicher Anschauung wirklich in der fortlaufenden Zeit in zusammenhängender Weise. “

„Zweitens ist für diese heilsgeschichtliche Zeitbewertung charakteristisch die Bezogenheit aller Punkte dieser Heilslinie auf die eine geschichtliche Tatsache der Mitte, die gerade in ihrer banalen Einmaligkeit heilsentscheidend ist: Tod und Auferstehung Jesu Christi.“ „Die

1 Zitiert nach: Immanuel Kant, Werke in sechs Bänden, hrsg. von Wilhelm Weischedel, Bd. II, Darmstadt 1956, 80/81.

2 Vgl. dazu auch Wolfgang Deppert über die Notwendigkeit der Aufspaltung des Zeitbegriffes in einen erkenntnislogischen und einen ontologischen Zeitbegriff. (W. Deppert, Zeit. Die Begründung des Zeitbegriffs, seine notwendige Spaltung und der ganzheitliche Charakter seiner Teile, Stuttgart 1989, 208ff.)

3 Oscar Cullmann, Christus und die Zeit. Die urchristliche Zeit- und Geschichtsauffassung. Zitiert nach der dritten, durchgesehenen Auflage, Zürich 1962. Zitate auf den S. 45f., 49, 60, 69. - Zu Cullmanns Werk vgl. auch Karl Löwith, Weltgeschichte und Heilsgeschehen. Die theologischen Voraussetzungen der Geschichtsphilosophie, Stuttgart ${ }^{3} 1953,168 \mathrm{ff}$., $225 \mathrm{ff}$. 
verschiedenen einzelnen Abschnitte der Gesamtlinie sind ständig von dieser Mitte her bestimmt, haben aber doch ihre zeitliche Eigenbedeutung."

Cullmann betont, daß ,urchristlicher Glaube und urchristliches Denken nicht ausgehen von dem räumlichen Gegensatz Diesseits-Jenseits, sondern von der zeitlichen Unterscheidung Ehemals-Jetzt-Dann. Damit soll nicht etwa gesagt sein, daß der eher räumliche Gegensatz von sichtbar und unsichtbar hier nicht bestehe. Es gibt im Neuen Testament einen unsichtbaren Himmel und eine sichtbare Erde; unsichtbare Mächte und Gewalten sind am Werk, während der Mensch nur die sichtbaren, durch ihre irdischen Organe ausgeführten Taten feststellt. Aber dieses unsichtbare Geschehen ist ganz und gar auch dem Zeitablauf unterworfen. Das Wesentliche ist nicht der räumliche Gegensatz, sondern die glaubensmäßige Unterscheidung der Zeiten."

Zeit ist also ,nach urchristlicher Auffassung nichts Widergöttliches, sondern das Mittel, dessen Gott sich bedient, um sein Gnadenwirken zu offenbaren. Die Zeit steht einerseits nicht im Gegensatz zu Gottes Ewigkeit, andererseits ist sie als gerade Linie, nicht als Kreis gedacht. Denn es wird von einem ,Anfang ${ }^{6}$ und einem ,Endec, einer d’@xý und einem $\tau \hat{\imath} \lambda$ os gesprochen. Sobald ,Anfang und ,Ende ${ }^{c}$ unterschieden werden, ist die Gerade die adäquatere Figur“".

Damit ist das Verhältnis von Zeit und Ewigkeit angesprochen. „Für das griechische Denken in seiner platonischen Ausprägung besteht zwischen Zeit und Ewigkeit ein qualitativer Unterschied ... Ewigkeit ist für Plato nicht etwa ins Unendliche verlängerte Zeit, sondern etwas ganz anderes: sie ist Zeitlosigkeit. Die Zeit ist für Plato nur das Abbild der so verstandenen Ewigkeit. Wie sehr das heutige Denken im Griechentum und wie wenig es im biblischen Christentum wurzelt, kommt uns zum Bewußtsein, wenn wir feststellen“, so Cullmann, „daß sogar bis in die weitesten Kreise christlicher Kirche und christlicher Theologie Zeit und Ewigkeit auf platonisch-griechische Weise unterschieden werden“. Für die biblische, urchristliche Zeitauffassung gilt, „daß die - nur als Attribut Gottes mögliche - Ewigkeit unendliche Zeit ist, oder besser gesagt, daß das, was wir ,Zeit' nennen, nichts anderes ist, als ein von Gott begrenztes Stück dieser gleichen unendlichen Zeitdauer Gottes. “

Das kurze Referat einiger Aussagen Cullmanns ${ }^{4}$ macht die konstitutive Rolle der Zeit und deren Besonderheit in der biblischen Zeitauf-

4 Sie ließen sich ergänzen u.a. durch Aussagen von Adolf Darlap, Zeit, „in der inkarnatorischen Existenz des Logos endgültig und bleibend , angenommen " " muß „als Erscheinung des Logos betrachtet werden, als eine wesentliche Dimension an 
fassung deutlich. Diese Zeitauffassung steht im Gegensatz zum Konzept einer „zyklischen“, kreisförmig geschlossenen Zeit, das selbst wiederum unterschiedliche Ausformungen erfuhr. So ist "mythische Zeit" „Zyklische Zeit"“, Zeit der „Ewigen Wiederkehr" ${ }^{\text {“5 }}$. Ein Vorbild findet sie im Kreislauf der Naturzeit. Von christlicher wie von mythischer Zeit ist die profane Zeit zu unterscheiden. Profane Zeit ist gekennzeichnet vor allem durch das Strömen, den Fluß, das Auftauchen von Gegenwart aus Vergangenheit in das Unbestimmt-Offene der Zukunft. Christliche und mythische Zeit legen Zäsuren in die profane, verwandeln sie. Von der profanen Zeit aus muß die ,heilige Zeit" - sei es die christliche, sei es die mythische - als Einbruch einer anderen Zeit erfahren werden.

Ein Werk der bildenden Kunst stellt sich - unter der hier erörterten Fragestellung - dar als Durchdringung von Zeit als "innerem Sinn“ die Gestaltung wie Rezeption entscheidend mitbestimmt - und „transsubjektiver" Zeit als Dimension des Thematischen, als christliche, mythische oder profane Zeit.

Ausgewählte Werke der Malerei des 15. und des 17. Jahrhunderts seien nach der Konstitution ihrer Zeitgestalt durch Farb- und Helldunkelkomposition kurz beschrieben ${ }^{6}$.

In Rogier van der Weydens Columba-Altar (entstanden um 1445/50, Alte Pinakothek München) erscheint der Bildgrund in einem dunklen, sich zum Horizont hin auflichtenden Blau. Es herrscht weder Nacht noch Tag - oder vielmehr beides. Trotz solcher die Naturzeit überhöhenden Synthese ist es das Blau Mariens, ein tiefes, strahlendes Ultramarinblau,

dem, was wird, wenn und indem der Logos selbst als solcher sich in der Andersheit des Nicht-Göttlichen und Endlichen zur Erscheinung bringt und zeigt. Darum ist Zeit in der HI. Schrift nicht als gleichmäßiges Weiterfließen einer letztlich leeren, nichtigen Selbstaufhebung des je jetzt Bestehenden gesehen, sondern immer schon strukturiert betrachtet: als einmalige und gerichtete Heilszeit, als Heilsgeschehen sowohl der Menschheit als ganzer wie auch des einzelnen, und endlich in wachsender offenbarungsgeschichtlicher Enthüllung als das in der Heilsgeschichte der Menschheit und des einzelnen in Gnade und Wort immer schon die Geschichte treibende und diese auf ihren letzten Sinn hin auslegende und sich selbst eschatologisch zeitigende eine und einzige Heilsereignis Jesus Christus." (In: Handbuch theologischer Grundbegriffe, hrsg. von Heinrich Fries, Studienausgabe, Bd. II, München 1963, 897.) Vgl. auch Darlap, in: Sacramentum Mundi, Bd. IV, Freiburg/Br. etc. 1969, Sp. 1423-1429.

5 Vgl. Kurt Hübner, Die Wahrheit des Mythos, München 1985, 142f. - Dort weitere Literatur.

6 Abbildungen der besprochenen Werke finden sich in den Katalogen der Alten Pinakothek, München, und des Metropolitan Museum, New York, sowie in umfangreicheren Monographien zu den betreffenden Künstlern. 
das den Blick zuerst auf sich zieht und ihn dann immer erneut auf sich versammelt. Das Blau der Maria der Anbetungsszene bindet an seinem zentralen Ort auch das Blau der Flügel an sich. In der Mitteltafel richten sich die Figuren von beiden Seiten nach innen, hin zum Christuskind als auf ihre gemeinsame Mitte. Nicht im Bildgrund versinnlicht sich das Absolute, sondern in der Unscheinbarkeit des Christuskindes, und die bildkünstlerische Komposition tut das Ihre, um diesem heiligen Ereignis gerecht zu werden. Von der Mitteltafel aus erschließt sich der linke Flügel, die „Verkündigung“, als heilsgeschichtliche Voraussetzung der Christusgeburt. Der weiße Saum im Gewand des rechten Königs verweist auf das Weiß des Verkündigungsengels, und zwar über die bezeichnende Differenz, daß das Weiß des Engels kühler, weil bläulich-verschattet wirkt und gerade darin seine Nähe zum Blau Mariens bekundet. An die verehrend-grüßende Gestalt dieses Königs können wir uns anschließen, mit ihm nähern wir uns der heiligen $\mathrm{Mitte}^{7}$, wie aus der Bildtiefe der Zug der heiligen Drei Könige zu Maria und Christus strömt, damit auch die Landschaft auf die heiligen Figuren hin orientierend. Der Bildgrund dient dem sakralen Geschehen, ist ihm nicht übergeordnet. Der rechte Flügel veranschaulicht mit der „Darbringung im Tempel“ Christi Weg in die Welt, aus der Geborgenheit bei der Mutter. Simeon, der das Christuskind aus Mariä Händen empfängt, ist gekleidet in ein Stahlblau, das dem Blau des Himmels verwandter ist als dem Mariens, und in ein strahlendes Rot, das die Rottöne der Mitteltafel und des linken Flügels ein letztes Mal erklingen läßt. Das Grün der Korbträgerin, mit dem der rechte Flügel einsetzt, nimmt dagegen das Wiesengrün verwandelt auf. Weit öffnet sich der Tempel nach rechts, in ein helles, klares Blau, in eine offene Zukunft des irdischen Schicksals Christi. Die Tempelarchitektur verweist aber auch auf das Mittelbild zurück und damit auf die dem Christuskind entgegenkommende Welt. Das ganze Heilsgeschehen verdichtet sich in der Bildzeit des Altares: die absolute Mitte im Christuskind des Bildzentrums, der Weg in seine irdische Zukunft in die rechts sich öffnende Weite, die absolute Mitte wiederum zeichenhaft, in dem im Dunkel sich verbergenden Kreuz der Bildmitte. Farb- und Helldunkelgestaltung tragen entscheidend bei zur Darstellung dieses Weges.

7 Vgl. M.B. McNamee, S.J., An Additional Eucharistic Allusion in Van der Weyden's Columba Triptych, Studies in Iconography, Vol. II, 1976, Northern Kentucky University 1977, 107-113. (S. 109: „... when early book illuminators and panel painters represented the procession of kings making their offerings to the Christ Child in Epiphany scenes, they would most probably have had in mind the traditional offertory procession of the Magi performed every year in the churches.") 
Hier ist nochmals an die zweite Besonderheit der christlichen Zeitauffassung zu erinnern, „die Bezogenheit aller Punkte der Heilslinie auf die eine geschichtliche Tatsache der Mitte ...: Tod und Auferstehung Jesu Christi." „Das chronologisch Neue, das Christus ... gebracht hat, besteht darin, daß für den gläubigen Christen die Mitte [des Heilsgeschehens] ... nicht mehr in der Zukunft liegt." Ausgangspunkt des biblischchristlichen Verstehens ist „die Mitte: von ihr aus erschloß sich der göttliche Heilsplan nach vorn und nach rückwärts ${ }^{\text {“8 }}$.

Die Bildkomposition entspricht dieser Zeitauffassung. In der unterschiedlichen Figurenbesetzung der linken und rechten Bildzonen und in den gegensätzlichen Bewegungsrichtungen rechts kann sie darüber hinaus eine grundlegende Differenzierung der heilsgeschichtlichen Bewegung wenigstens verhalten andeuten: die eine Bewegung der Heilsgeschichte nämlich „geht von der Vielheit zum Einen: das ist der Alte Bund“. Sie verläuft im Sinn „einer progressiven Reduzierung: Menschheit - Volk Israel - Rest Israels - der Eine, Christus. Bis dahin tendiert die Vielheit auf den Einen hin, auf Jesus Christus, der als Christus Israels zum Erlöser der Menschheit, ja der Schöpfung wird. Hier ist die Heilsgeschichte in ihrem Zentrum angelangt." Die andere Bewegung „geht von dem Einen zur Vielheit, das ist der Neue Bund“. „Der Weg geht ... jetzt von Christus zu denen, die an ihn glauben ... Er führt zu den Aposteln, zur Kirche ... und weiter zur erlösten Menschheit im Gottesreiche und zur erlösten Schöpfung des neuen Himmels und der neuen Erde. “9

Damit ist der allgemeine Rahmen christlicher Zeitauffassung benannt. Jedes Werk christlicher Thematik bringt eine je eigene Verwirklichung dieses Zeitverständnisses.

Eine der Inkunabeln der niederländischen Malerei, Robert Campins um 1425 gemalter Verkündigungsaltar (The Cloisters, Metropolitan Museum of Art, New York), zeigt eine verwandte Zeitstruktur. Im Weiß des Engels sammelt sich das Licht, es beleuchtet ihn und strahlt von ihm aus. Er bildet die Einleitung der Figuralkomposition und der sie rahmenden Räume. (Das göttliche Licht selbst, das in dünnen goldenen Strahlen durch das linke Fenster dringt, verbirgt sich eher, wie auch das ganz kleine Christuskind in diesen Strahlen, das schon das Kreuz trägt, sich dem Blick nahezu entzieht.) Das Gelb des Engelsflügels nimmt das Licht in die Buntfarbigkeit auf, weißliche Aufhellungen gleiten im Mariengewand in kostbares Karminrot über. Samtgrün in Mariens Tasche,

8 Cullmann (wie Anm. 3) 46, 84, 104.

9 Cullmann (wie Anm. 3) 112. 
ins Dunkle sinkendes Kobaltblau begleiten es. Mildes Ockerbraun füllt die Tischplatte, die in ihrer polygonalen Brechung wie auf Maria hin zu rotieren scheint. Ein schmaler Dunkelsaum umgibt die Tischkante und sinkt unter dem Tisch in eine transparente Dunkelhöhle. In transparente Dunkelheiten versinken auch der Fußboden und die Ofenwand. Von den weißgrauen und -bräunlichen Zimmerwänden heben sich die schwärzlichbraunen Fensterläden ab. Ihre Dunkelrhythmik nimmt die Rückwand der Werkstatt des heiligen Joseph auf dem rechten Flügel auf. Dort vertieft sich die Dunkelheit insgesamt, zugleich aber weitet sich der Bildraum in die von einem hellen, milchigen Himmel überspannte Stadtansicht. Grauviolett gebrochen ist das Gewand Josephs. Rot in seinen Ärmeln, Blau in seiner Mütze sind nur mehr Begleitfarben. Ist der Bildraum der Mitteltafel eng um die heiligen Figuren gespannt und damit auch die Bildzeit ganz aus dem heiligen Geschehen entfaltet, getragen auch von der Herablassung des aus sich strahlenden Lichtes in die Unterschiedenheit der Buntfarben und in die alle Farben umfangende Dunkelheit, so gewinnt der Bildraum im rechten Flügel an Eigenleben, und zugleich entspannt sich die Bildzeit in die Mannigfaltigkeit irdischer Tätigkeiten in Nähe und Ferne. Der linke Flügel aber ist hier Ort des Nebenmotivs, zeigt die Figuren der Stifter in schwarz-dunklen Gewändern, in halbdunklem Hofraum. In ihren Haltungen lassen die Stifter die Engelsfigur nachklingen, ahmen sie nach.

Auch in Dieric Bouts' Gefangennahme Christi (Alte Pinakothek München) wird die Bildzeit strukturiert durch die Farb- und Helldunkelgestaltung. Die Folgeordnung setzt ein, nach den Helligkeits- wie nach den Raumwerten, mit dem leuchtenden, mondhaften Gelb von Malchus, dem Petrus das Ohr abhauen will. Die roten Säume seiner Jacke binden ihn an den zinnoberroten Mantel Petri. Mit dem sehr dunklen Blau des Petrusmantels spannt sich die Bewegung der Figurengrupppe, gleichsinnig dem emporgerissenen Arm Petri, aufwärts zum etwas grünlicheren Blau des Nachthimmels. Der Kontrast von farbigem Licht und farbigem Dunkel bildet so das Eingangsmotiv der Figurenentwicklung. Im Komplementärkontrast zum Petrusrot steht das kühle Grün des Judas, der Christus küßt. Der Zinnoberton aber führt mit den Armen des Christus wegziehenden Schergen nach rechts, zum schließenden Rot im Mantel des Soldaten rechts. Das dünne, weißlich aufgehellte Karminrot seiner Hose nimmt den Farbton der Malchushose auf, das dunkle Blau seiner Jacke den des Petrusgewandes - aber die Farben des Schergen erscheinen durch Form- und Farbbindungen dynamisiert, durch Aufteilung in kleinere Bezirke beim Blau, durch gleitende Bewegung zu Graurosa, 
Lachsrosa und Goldbraun beim Rot. Die Rottöne bindet wiederum ein Grün, ein anderes, gelblicheres, im Mantel des auf Christus eindringenden Schergen. Dem Wirbel dieser farbig eng miteinander verknüpften, in ihren Bewegungsrichtungen aber gegeneinander kontrastierten Schergen ist Christus wehrlos preisgegeben und bleibt ihm zugleich doch entrückt. Das bleiche, grau- und violettonige Blau seines Passionsmantels löst sich aus dem Zusammenhang sowohl der Bunt- wie der Neutralfarben, isoliert sich zudem durch seine nur ihm eigene leuchtende mittlere Helligkeit. Innerhalb der rechten Figurengruppe entsteht eine unstabile Lichtform aus Weißflecken von wehendem Tuchbausch, Ärmel, Turbanen, Fackellicht und Mond. Sie öffnet sich nach oben rechts, verweist damit auf den Fortgang des Geschehens, Christi Vorführung vor dem Hohenpriester. Mit einer figural verdichteten Lichtform, dem Gelb des Malchus, setzt die Bildbewegung ein, mit einer punkthaft aufgelösten endet sie, eine eindeutig gerichtete, aber durch Zäsuren zerrissene und damit expressive, das Thema interpretierende Bildzeit veranschaulichend. Sie konfrontiert die dramatische Zeit menschlicher Handlungen mit der stillen Gegenwart Christi, die sich zugleich entzieht.

In Rubens' Malere ${ }^{10}$ lassen sich Formen christlicher und mythischer Zeit, den jeweiligen Themen entsprechend, klar unterscheiden. Ihre Träger sind Körper-, Farb- und Helldunkelkomposition.

Bei seinem Jüngsten Gericht, entstanden wohl kurz nach 1614 (aufbewahrt, wie auch die folgenden Werke von Rubens, in der Alten Pinakothek München), setzt die Folge der Inkarnat- und Gewandfarben ein mit dem grau und blaugrau verschatteten Orangebraun des vornübergebeugt Auferstehenden in der unteren Bildmitte und dessen, der die Grabplatte hochstemmt. Darauf folgt das fahlgelbliche Inkarnat des Mannes in der linken unteren Bildecke. Mit der hinter der Grabplatte aufstrebenden jungen Frau, ausgeprägter noch mit der Kauernden darüber, heben die „allfarbigen“ Inkarnate an, auf Rosaton gestimmt, mit gelblichen Lichtern, blaugrauen Schatten und zinnoberbräunlichen Reflexaufhellungen in den Schatten. Darüber steigen in Gewändern die Grundfarbtöne auf, aus der Dunkelheit am linken Bildrand ein braunrotes Engelsgewand, darüber ein lichtblaues. Hier klingt erstmals der Rot-Blau-Akkord auf, der dann machtvoll in Christus und Maria sich

10 Vgl. dazu auch Verf., Versuch über die Farbe bei Rubens, in: Erich Hubala (Hg.), Rubens. Kunstgeschichtliche Beiträge, Konstanz 1979, 37-72. - Verf., Helldunkel und Konfiguration bei Rubens, in: Frank Büttner, Christian Lenz (Hgg.), Intuition und Darstellung. Erich Hubala zum 24. März 1985, München 1985, 105-116. 
steigert. Dazwischen schließen sich Engelsfarben zur sekundären Trias zusammen, zum Akkord von Orangerosa, Grün und zart lilatonigem Graublau. Darüber Petrus in mildem Sandgelblich, Johannes in Korallrot und, wie schon erwähnt, Maria in lichtem, festem Blau. Zur Totalität entfalten sich die Farben auf der Spitze der Menschen- und Engelsäule, zur Trias der Grundfarben vereinen sie sich in den Gewändern Petri, Mariä und Christi und lassen doch, durch einen Rahmen weißgelblichen Lichts, dem Rot des richtenden Christus seine Einzigartigkeit. Rechts neben Christus klingen nochmals, zarter, Buntfarben auf. Dann aber fahren dunkelgraue Wolkenbahnen dazwischen, trennen in scharfer Zäsur von den Seligen und Heiligen die Verdammten. In den Engelsgewändern erscheinen nochmals Mischfarben, dann nur noch fahles Grau, Bräunlich, Grünlichgrau, Grau- und Orangebraun in den Inkarnaten, einige wie angesengt vom Rotbraun der Hölle. In Figuren- wie Farbenfolge eine Zeit der Entscheidung, der Unterscheidung, des Gerichts versinnlichend, in heftiger Zäsur Kontinuität und Wiederkehr vereitelnd.

Von Dunkelzäsuren ist auch des Künstlers Niederlage Sanheribs (gemalt um 1612/14) geprägt. Kühlgelbliches Licht bricht aus dem Himmel ein und zerreißt die Finsternis. In zwei Lichtzentren sammelt es sich auf den irdischen Gestalten, in der Mittelgruppe um Sanherib und in der Masse der kopflos fliehenden Krieger rechts. Ein drittes, ein Nebenzentrum, bildet der nackte Leichnam links unten. Diese Lichtkomplexe werden durch Dunkelsäume entschieden getrennt. Ein einmaliges, durch göttlichen Eingriff bewirktes Geschehen wird dargestellt, unwiederholbar, aus dem Naturkreislauf entrückt. Aber die Strahlen des Lichts ziehen an Sanherib vorbei, treffen die blindlings Flüchtenden, auf die auch der herabstürzende rotgewandete Engel zielt. Das Bild hat zum Gegenstück die Bekehrung Pauli, die sich im Courtauld Institute, London, zur Princes Gate Collection gehörig, befindet. Etwas von Einsicht in die Macht eines Höheren wird auch Sanherib zuteil, Erfahrung eines Lichtes, das nicht das des Blitzes, das der Naturenergie ist, Erfahrung damit auch einer anderen Zeit, die in die Naturzeit einbricht ${ }^{11}$.

11 Vgl. dazu: Hans Gerhard Evers, Rubens und sein Werk, Brüssel 1944, 260: Rubens hat „das Bild als Gegenstück zur ,Bekehrung Pauli‘ gemalt, in fast mittelalterlicher Weise als Vorbedeutung und Erfüllung aus Altem und Neuem Testament, und hat sie aneinander angeglichen, indem er sie beide zu Lichterscheinungen, zu Blendungen machte .... “. - Dazu auch: Corpus Rubenianum Ludwig Burchard, Part III. R.-A. d'Hulst and M. Vandenven, Rubens. The Old Testament, London 1989, 150 154. 
Rubens' Amazonenschlacht (entstanden um 1615) dagegen kann als Inbild einer mythischen Schlachtdarstellung gelten, in der Menschen und Tiere als Glieder eines elementaren Naturkreislaufs erscheinen. In seinem Graublauton nimmt der Griechenreiter links oben das Blau des Himmels auf, das Lachsrot der Amazone neben ihm ist wie gefärbt von Feuerschein, der auch die bräunlichrote Brandwolke beleuchtet, im Ockergelb des Pferdes, das der griechische Schwertkämpfer reitet, wird ein Sandton aufgenommen, wie Erdtöne im Braun der Amazonenpferde. Volles, kraftstrotzendes animalisches Leben geht unter, geht ein in abgrundhaftes Dunkel. (Das Einleitungs-Nebenmotiv in der linken unteren Bildzone, einsetzend mit der rotgewandeten Amazone auf weißbraun geflecktem Pferd, verdichtet das Bildgeschehen auf seine Quintessenz.) Kein Kampf ist dargestellt im Widerprall zweier Gegner, dessen Entscheidung noch offen stünde, sondern eine naturhafte Verkettung von Angriff und Untergang, höchstes Leben, das sich erfüllt im Untergang als Bezeugung einer naturhaft-kosmischen Notwendigkeit. Leben geht ein in die Dunkelheit des Wassers, den Ursprung allen Lebens. Die Amazonen und ihre Pferde stürzen ins Dunkel, aber auch die Griechen werden in der Ferne von Dunkelheit eingeholt. Über ihnen tut sich dann wieder das Licht auf, aber ein gelbliches Licht von der Farbe des Wassers! Licht und Wasser sind eins wie Dunkel und Wasser. Leben triumphiert, steigert sich in der Gewißheit des Todes, der kein letzter Untergang ist. Die eindeutige Richtung des Schlachtgeschehens mit ihrem Einsatz von links wird überformt von einer Helldunkelrhythmik, die ihren Schluß rückbindet in ihren Anfang, aus der Dunkelheit der Wolke sich in die Schattenzonen der Pferde und Menschen verleiblicht, in kreisende Abgründe des Wassers sich öffnet, in der Dunkelheit der Ferne wieder Bezug nimmt zur lichteren Dunkelheit der Wolke. Diesem Helldunkelrhythmus gliedert sich auch die Farbgestaltung ein. Die mittlere Dunkelheit des Himmelsblaus, das den Beginn der figuralen Entfaltung foliiert, entläßt aus sich die Helligkeiten wie die Dunkelheiten. Mit einem hellen Pferd setzt das Kampfgeschehen ein, mit einem dunklen findet es rechts seinen figuralen Schluß, die kreisenden Dunkelheiten weiten die Rhythmik ins Überfigurale, und darin schließen sich Lichtzone und Lichtzone, Dunkelzüge und Dunkelzüge im Kreisgang zusammen - zur Versinnlichung einer kosmischen, zyklisch geschlossenen und sich wiederholenden Zeit.

Auf andere Weise wird in seinem Trunkenen Silen naturhafte Zeitlichkeit anschauliche Gestalt. (Das Werk wurde um 1617/18 zunächst als Halbfigurenbild konzipiert und um 1626 durch Anstückungen an den 
seitlichen und am unteren Bildrand erweitert.) Diese Zeitlichkeit wird getragen von den Bewegungen der mächtigen Leiber. Die Satyrmutter mit ihren beiden Säuglingen bildet das Eingangsmotiv. Ganz Dumpfheit, ganz Hingabe an den Genuß und das Leiden am Genuß, gehört die Gruppe der Erde an, aus deren Farbton die Farbe des Fells aufsteigt und daraus, schon durch Licht verklärt, die Farbe des Inkarnats. Im Fell des rechten Säuglings sinkt die Farbe wieder in den Erdton zurück. Aus diesem dünnen, streifigen Ockerbraun erwächst dann, etwas bräunlicher, der Kopf der geduldigen Ziege. Ihr Fell wird lichter, grauer. Die Farbe gleitet über in die des Bocks, der sie bespringt - ohne Gier, ernst, als Verkörperung des heiligen, sich selbst erneuernden Lebens. Wie segnend legt der nackte Junge die Hand auf die Stirn des Tieres. Staunend blicken der alte Bauer und die alte Bäuerin auf die säugende Satyrmutter, dieses Symbol des wiederanhebenden kraftvoll-dumpfen Lebens. Das Bild verkündet den Triumph des Tiere, Menschen und Zwischenwesen umfassenden Lebenskreislaufs, der das Alter zurückbindet an die Entstehung des Lebens. Eine junge Schöne läßt sich von einem Alten bedrängen. Ein lachender Neger hält den trunkenen Silen, unsanft seine Fleischlichkeit aufweisend, ein Satyr stützt ihn an seiner Rechten. Aber Silenos bedarf der Stütze nicht eigentlich. Er selbst ist ja Inbild des in sich kreisenden Lebens, mit dem Zentrum in der Kreisform des Bauches. Um diese Kugel scheint die Bildbewegung zu kreisen. Silenos torkelt und wird doch nicht fallen. In den Strahlen des weißgelben und weißgrauen Felles wie in den aufglühenden Reflexen des zinnoberroten Mantels verliert der schwere, ungefüge Leib sein Gewicht. Wie Ringe legen sich die Begleitfiguren um den inneren Ring, die braune Dunkelheit des Negers rechts, die blaue Dunkelheit der freundlichen Alten links, die den Krug schon wieder bereithält. Sie ist von links beleuchtet, ihr weißes Kopftuch sammelt dies von außen kommende Licht, Silenos aber scheint aus sich selbst zu strahlen. Die Helligkeit seines Inkarnats weist auf die Helligkeit des Inkarnats der Satyrmutter, das Haupt- und Schlußmotiv weist zurück auf den Anfang, zyklisch die Wiederholung des Kreislaufes ermöglichend.

In eine andere Welt der Zeitkonstitution führt uns die Kunst Rembrandts. In seinem gedankenreichen Rembrandt-Buch handelte Georg Simmel ausführlich von der Darstellung der „Lebensvergangenheit" bei Rembrandt ${ }^{12}$. „Die Rembrandtsche Menschendarstellung läßt uns jeweils

12 Georg Simmel, Rembrandt. Ein kunstphilosophischer Versuch, Leipzig ${ }^{2} 1919,41 f$., 44, 43f., 45 . 
eine Totalität des Lebens erschauen, trotzdem diese begrifflich und als äußerliche Realität in das Nacheinander von Vergangenheit und Gegenwart geformt ist. Wir sehen eben den ganzen Menschen und nicht einen Augenblick seiner, von dem wir dann erst auf frühere Augenblicke schlössen; denn das Leben ist unmittelbar gar nichts anderes, als die Gegenwart werdende Vergangenheit, und wo wir das Leben wirklich sehen, läßt uns ein bloßes Vorurteil behaupten, daß man nur den starren Punkt der Gegenwart sehe.“ Bei Rembrandt wird ,jedes Gebilde als ein in der flutenden Rhythmik von Leben, Schicksal, Entwicklung gewordenes oder werdendes erschaut ...: es ist sozusagen nicht diese jetzt erreichte Form, die Rembrandt vorträgt, sondern das gerade bis zu diesem Augenblick gelebte, von ihm her gesehene Gesamtleben ...".

Solche Bestimmungen verband Simmel mit den Aussagen zum Vorgang des Sehens, die in einem prinzipielleren Sinne wichtig sind und deshalb ausschnitthaft zitiert werden sollen: „Das Sehen ... hat, als Vorgang des Lebens, an dessen allgemeinem Charakter teil: daß dafür die Scheidung von Vergangenheit, Gegenwart und Zukunft nicht so gilt, wie die grammatikalisch-logische Schärfe zu fordern scheint. Erst nachträgliche Grenzstriche bringen diese Alternative an die kontinuierliche Lebensströmung heran ... Wo wir Leben wahrnehmen und nicht einen erstarrten Querschnitt, der nur einen Inhalt, aber nicht die Funktion des Lebens als solchen bietet, nehmen wir stets ein Werden wahr (sonst könnte es nicht Leben sein), nur wo die eigentümliche Fähigkeit in Funktion tritt: das Jetzt in der Kontinuität eines zu ihm sich streckenden Ablaufs anzuschauen, haben wir wirklich Leben gesehen." Und nochmals: „... wo wir Leben wahrnehmen, (ist) die absolute Scheidung eines hart isolierten Jetzt gegen ein ausgedehntes Vergangenes Sache einer intellektuellen Reflexion; in Wirklichkeit nehmen wir zunächst und unmittelbar eine zeitlich erstreckte, gar nicht in Momente auseinanderfallende Einheit wahr ...".

Das Medium der Darstellung von Lebenstotalität bei Rembrandt ist das Helldunkel. Helldunkelfarbe ist werdende, dynamisch gespannte Farbe, ist Übergang, ist Auftauchen und Versinken. Die Formen, die im Helldunkel entstehen, werden von dessen Rhythmik mitbewegt.

Das Antlitz auf Rembrandts Selbstbildnis von 1660 (The Metropoli$\tan$ Museum of Art, New York) scheint von links beleuchtet - aber das Licht steigt zugleich aus dem Dunkel selbst empor. Der Hintergrund wird im Bewegungsstrom des Antlitzes mitbewegt, steigt wolkig links empor, hellt sich über dem Barett auf. Die Falten des Gewandes, die Falten des Antlitzes, die Haare, der Kragen nehmen in ihrer Rhythmik 
der Aufhellungen und Verdunkelungen an dieser Gesamtbewegung teil, am Aufstieg links und Sinken rechts. Die Rhythmik, von der Simmel sprach, ist eine gerichtete, wie auch die Rhythmik des Lebens, die Lebensbewegung selbst, gerichtet ist. Auch die Konturführung der Mütze fügt sich dieser Bewegung ein, emporsteigend links, sich senkend rechts. Aber von der Farbe dieser Mütze wird die Bewegung nicht mitvollzogen. Mit ihrem homogenen, unbewegten Braunschwarz vergegenwärtigt sie ein Absolutes, Endgültiges, in sich Abgeschlossenes, das das Hinfällige, von Vergangenheit Gezeichnete des Antlitzes übersteigt.

Max Raphael formulierte in seiner Beschreibung dieses Bildes: Das Schwarz „bildet die absolute Ruhe in der permanenten Bewegung, die völlige Unbestimmtheit im Vibrieren zwischen Bestimmtheiten; das Schwarz transzendiert das Licht, den Raum, die Immanenz ... Es fügt so dem Porträt diejenige Dimension hinzu, die nicht porträtiert werden kann: die des prozeßhaften Seins, welches das Sein eines Nichtseienden, die Fülle eines Unbestimmten und Unbestimmbaren darstellt ..."13.

Ganz verhalten aber nimmt dieses Schwarz dennoch teil an der Helldunkelrhythmik des Ganzen: an der oberen Spitze des Baretts lichtet es sich ein wenig, öffnet es sich in das Braun. Nach rechts hin fließt es über den Mützenkontur hinweg in die Dunkelheit des Grundes.

Vor allem aber nehmen die Pupillen das Braunschwarz der Mütze auf und verdichten es. Simmel sprach vom "raumlosen Blick“ in Rembrandts späten Porträts und bezeichnete als ein Charakteristikum dafür das „Verlöschen des Glanzlichtes im Auge“. „Dieses Immer-Weitersehen" ist ihm „ein Symbol der Lebendigkeit, die sich an keinem einzelnen Inhalt, auch an keinem subjektiv-individuellen genugtut, sondern unter oder über jeden Weg ins Unendliche flutet - womit sich eben nur ausdrückt, daß sie überhaupt nirgends hin flutet, weil sie nicht von einem terminus ad quem abhängig ist. Im begrifflichen Sinne, der sich an einem Gegenstande orientiert, mag dieser Blick irgendwie unbestimmt wirken, im Lebenssinne ist er etwas durchaus Sicheres."

Er ist deshalb etwas Sicheres, weil sich in ihm ein Wissen des dem Leben innewohnenden Todes bekundet (womit auch die schwerverständliche Rede Max Raphaels vom „Sein eines Nichtseienden“ ihren Sinn gewinnen mag). „... ein Punkt der Zukunft, der überhaupt das Leben erst zu einer Ganzheit macht, und zwar gerade indem er es abbricht, wohnt in allen tiefsten Rembrandtporträts: der Tod.“ „... das Moment

13 Max Raphael, Die Farbe Schwarz. Zur materiellen Konstituierung der Form. Mit einem Nachwort von Bernd Growe, hrsg. von Klaus Binder. Frankfurt/M., Paris $1983,70$. 
des Todes, das in allem Lebendigen enthalten ist, (scheint mir)“, schrieb Simmel, ,in dem Bilde des Menschen, wie Rembrandt es faßte, nachdrücklicher und herrschender fühlbar zu sein, als irgendwo sonst in der Malerei ..." 14 .

Ich wende mich religiösen Bildern Rembrandts zu und betrachte seinen in der Münchner Alten Pinakothek aufbewahrten Passionszyklus. Licht wird hier als Medium von Zukunft und Gegenwart, Dunkel als Medium von Vergangenheit erkennbar, und zwar in einer je anderen Modifikation.

Die Kreuzaufrichtung (entstanden gegen 1633) zeigt Christus herausgestrahlt aus einer umgebenden Finsternis. Kein natürliches Beleuchtungslicht bekundet sich hier, vielmehr eine Lichtform, die zwischen dem nach rechts oben gereckten, lichtversammelnden Leib Christi und dem nach links unten orientierten Lichtreflex in der Rüstung des das Kreuz aufrichtenden Soldaten ausgespannt ist. Diese energetisch gespannte Lichtform ist „Wesenslicht" des Geschehens, es offenbart das Ereignis in seiner zeitlichen "Quintessenz".

Bei der Kreuzabnahme (wohl 1633 gemalt) dagegen bildet das herausleuchtende Licht eine dynamische Form, die gleichsam nach unten „abtropft", hilflos, ohnmächtig den heiligen Leib ins Dunkel, in die Unbestimmtheit und Unfaßbarkeit, sinken läßt. Über alle naturzeitliche Konkretion hinaus ist das Licht dieser Bilder thematisch bestimmt, hebt als „Offenbarungslicht" das heilsgeschichtlich-zeitliche Geschehen in eine über dem empirischen Zeitfluß stehende zeitlich gespannte Gegenwart.

In der Grablegung Christi (1636/39) wandert der Blick zwischen zwei Lichtzentren, dem naturzeitlich bestimmbaren Licht der untergehenden Sonne und einem Licht unbestimmbarer Herkunft, das Christus beleuchtet, wohl eine Erscheinungsform des von Wolfgang Schöne freilich nicht sehr glücklich - so benannten ,indifferenten Leuchtlichts"15. Die Kerze, die der Mann nahe dem linken Bildrand in Händen hält, kann nicht Quelle dieses Lichtes sein. Das Licht der scheidenden Sonne erscheint dynamischer, wächst an, bricht sich hart an der Dunkelkante des Felsens, das Offenbarungslicht, das um Christus und seine Begleiter - von diesen kaum bemerkt - strahlt, wirkt demgegenüber als ein „stehendes“ Licht, verliert rasch an Kraft, dringt nicht tief in die umgebende Dunkelheit ein. Die Bewegung der Menschen aus der Finsternis

14 Simmel (wie Anm. 17) 127, 89f.

15 Vgl. Wolfgang Schöne, Uber das Licht in der Malerei, Berlin 1954, 112 und passim. 
in das Licht ist stärker als die des Lichtes in das Dunkel. So ist hier das Offenbarungslicht Licht einer überzeitlichen Gegenwart der Stille und des Abschieds.

Ganz anders die Lichtkomposition der Auferstehung Christi (1639): Licht bricht ein, der Engel öffnet den Weg in die himmlische Zukunft, wirft die Wächter zurück ins Dunkel, in die Vergangenheit des todverfallenen Lebens. Helldunkel ist hier Vergegenwärtigung des Kampfes zwischen göttlicher, lichthafter Zukunft und irdischer, dunkler Vergangenheit. Christus, noch halb vom Dunkel und vom Tode umfangen, erwacht in seine Zukunft und absolute Gegenwart.

Eine komplexere Zeitgestalt bestimmt die Himmelfahrt Christi (1636). Aus ihrer Dunkelheit und Vergangenheit wenden sich die Apostel aus der Tiefe ins Licht, in ihre Zukunft. Der Zeithorizont der Gegenwart wird anschaulich im Horizont, der die Betrachterperspektive fixiert, etwa in der Höhe der Wolke, auf der Christus steht. Er ist aber nur Übergang in die aufwärtsführende Bewegung, in das Empor der Himmelfahrt, an der wir selbst von ferne teilhaben dürfen. Der Saum und der Faltenkamm des Gewandes Christi erzittern im Schwung dieses Aufstiegs, nach oben hin aber wird die Bewegung immer stiller, verklingt in der Kreisform der Gloriole um den Heiligen Geist als anschaulichem Symbol des Bleibenden, der Ewigen Gegenwart, die aber Zukunft ist der Menschen wie des auffahrenden Christus.

Christus aber erstrahlt im hellsten Licht. So macht dies Bild an der Figur Christi die zeitliche Spannung zwischen dem "schon erfüllt" und „noch nicht vollendet", die Spannung zwischen Gegenwart und Zukunft sichtbar, die für christliches Zeitverständnis konstitutiv ist: Ich zitiere nochmals Oscar Cullmann: „Die Herrschaft Gottes über die Zeit, wie sie einerseits in Prädestination und Präexistenz, andererseits im Christusgeschehen selber offenbar wird, bedeutet nichts anderes, als da $\beta$ er, der Ewige, über die ganze Zeitlinie in ihrer unendlichen Ausdehnung verfügt, daß daher im Handeln Christi jeweils die ganze Linie in entscheidender Weise beeinflußt wird, und daß im zentralen Christusgeschehen des Inkarnierten, das die Mitte jener Linie darstellt, nicht nur alles Vorangehende erfüllt, sondern auch alles Zukünftige entschieden wird." Diese Zeitauffassung bestimmt das Verständnis des Gottesdienstes. In der Mahlfeier ist „Christus unter den Seinen gegenwärtig“, zugleich bezieht sich diese Mahlfeier sowohl ,rückwärts auf das Todesmahl des historischen Jesus und die Ostermahlzeiten des Auferstandenen mit seinen Jüngern, und vorwärts auf das Ende, das ja im Bilde des messianischen Mahles schon im Judentum vorgestellt wird“. Diese Zeitauffassung be- 
gründet auch das Verständnis der individuellen Heiligung des Glaubenden: „Es ist das Grundmotiv aller neutestamentlichen Ethik, daß der Mensch auf Grund des heiligen Geistes im Glauben an das von Christus vollbrachte Werk das ist, was er erst in Zukunft sein wird, daß er schon sündlos, schon heilig ist, was doch erst in Zukunft Wirklichkeit wird"16.

Rembrandts Kunst macht in besonderer Weise diese Spannung und Verdichtung der heilsgeschichtlichen Zeitdimensionen, die Spannung zwischen dem „schon erfüllt" und dem „noch nicht vollendet“" sichtbar. Es gelingt ihr dies durch eine neue Art der Versenkung der „inneren“ Zeit in die biblisch-christliche Zeit.

Bei der Anbetung der Hirten (1646, Alte Pinakothek München) schließlich kann alles, was über die „Lebensvergangenheit“ und die „Ganzheit des Lebens" bei Rembrandts Selbstbildnissen angedeutet wurde, in die religiöse Thematik verwandelt, wiedergefunden werden. Hier sammelt das Licht, es strahlt nicht aus. Aus dem Dunkel kommen die Menschen zum Licht. Es ist die Größe Rembrandts, daß er den Weg des Menschen, unseren Weg, zum Licht, zu unserer Zukunft mitdarstellt. Aus dem verdichteten Dunkel ihrer Lebensvergangenheit drängen die Menschen zum Licht, in seiner Stille und Ruhe findet ihr Leben seine Ganzheit, in einem Licht, das Zukunft verheißt und Ewige Gegenwart und das auch die Unfaßbarkeit des Dunkels, des Todes noch trägt.

Die Gegenüberstellung einiger Werke von Rubens und Rembrandt führt die Betrachtung auf die unterschiedliche Rolle des „Plastischen“ in der Malerei.

Zwar ist Integration des Plastischen in die Malerei ein Charakteristikum aller neuzeitlichen Gemälde ${ }^{17}$, doch darf insbesondere diejenige des Rubens als eine im ausgezeichneten Sinne ,plastische Malerei“ angesprochen werden. Auch darin erweist sich ihre besondere Nähe zu Themen der klassischen Mythologie ${ }^{18}$.

16 Cullmann (wie Anm. 3) 77f., 79, 80.

17 Vgl. Theodor Hetzer, Vom Plastischen in der Malerei, in: Hetzer, Aufsätze und Vorträge, Bd. II, hrsg. von Gertrude Berthold, Darmstadt 1957, 131-169 (erstmals publiziert 1938).

18 Otto von Simson schreibt: „Wie kein anderer Maler vor ihm, nicht einmal Tizian, läßt uns Rubens die Gottheiten der Klassischen Antike hier [im Bild „Krieg und Frieden"] nicht als die üblichen Allegorien erleben, als die eine lange Tradition sie gedeutet hatte. Venus als Göttin der Liebe nährt hier und beschützt zugleich alle Früchte des Friedens ... Keine andere Gestalt des antiken Mythos ist so bezeichnend für ihn“. (Politische Symbolik im Werk des Rubens, in: E. Hubala [wie Anm. 10] 9.) 
Aufschlußreich ist es, für die Erörterung der hier zu behandelnden Fragestellung Hegels Ästhetik heranzuziehen, verbindet diese doch, wie kaum eine andere, thematische Gesichtspunkte mit solchen der künstlerischen Gestaltung.

In Hegels „Vorlesungen über die Ästhetik“ heißt es: „Die Skulptur im allgemeinen faßt das Wunder auf, daß der Geist dem ganz Materiellen sich einbildet und diese Äußerlichkeit so formiert, daß er in ihr sich selber gegenwärtig wird und die gemäße Gestalt seines eigenen Innern darin erkennt."

So wird die Skulptur zur Kunst des klassischen Ideals, zum „Mittelpunkt der Klassischen Kunst": „Das Geistige in dieser vollendet selbständigen Beschlossenheit des in sich selber Substanziellen und Wahren, dies störungslose unpartikularisierte Sein des Geistes ist das, was wir die Göttlichkeit nennen, im Gegensatz gegen die Endlichkeit, als das Auseinandergehen in das zufällige Dasein, in die Unterscheidung und veränderliche Bewegung. Die Skulptur hat nach dieser Seite hin das Göttliche als solches darzustellen in seiner unendlichen Ruhe und Erhabenheit, zeitlos, bewegungslos, ohne schlechthin subjektive Persönlichkeit und Zwiespalt der Handlung oder Situation. Und geht sie nun auch zur näheren Bestimmtheit des Menschlichen in Gestalt und Charakter fort, so muß sie auch hierin nur das Unveränderliche und Bleibende, die Substanz dieser Bestimmtheit auffassen und nur diese, nicht aber das Zufällige und Vorübereilende sich zum Inhalt wählen ..." 19 .

Wird in dieser Aussage auch Hegels Zeitbedingtheit, sein klassizistischer Begriff der griechischen Klassik, erkennbar, so kann aus ihr doch zugleich die Differenz zwischen griechischer Skulptur und deren Renaissance, deren Wiederaufleben in der Kunst der Neuzeit (die sich ja häufig auf hellenistische Werke berief) abgeleitet werden.

All solcher Unterschiede unerachtet, eine ,selbständige Beschlossenheit des in sich selber Substanziellen“ ist Wesensmerkmal auch der „plastischen“ Darstellung der Götter bei Rubens. Für die nachantiken, die ,romantischen“ Künste aber steht nach Hegel, im Bereich der Bildenden Künste , die Malerei im Zentrum. In der Malerei nun ist es „das Innere des Geistes, das sich im Widerschein der Äußerlichkeit als In-

Die unterschiedliche Nähe neuzeitlicher Künstler zu bestimmten Göttergestalten der Antike wird damit angedeutet.

19 Hegel: Vorlesungen über die Ästhetik, Jubiläumsausgabe, hrsg. von Hermann Glockner, Bd. 13, Stuttgart 1928, 365, 376, 368. - Zu Hegels Begriff der Plastik vgl. Dieter Rahn, Die Plastik und die Dinge. Zum Streit zwischen Philosophie und Kunst, Freiburg/Br. 1993, 158-205. 


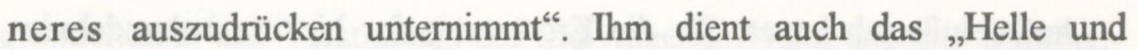
Dunkle“. Hegel schreibt: „In der Malerei ... gehört das Helle und Dunkle mit allen seinen Gradationen und feinsten Übergängen selber zum Prinzip des künstlerischen Materials und bringt nur den absichtlichen Schein von dem hervor, was Skulptur und Baukunst für sich real gestalten ....". Und weiter, nun über bloße Beleuchtungsphänomene hinausgehend: „Das Licht nämlich ... bezieht sich auf das ihm Andere, das Dunkle. In diesem Verhältnis bleiben jedoch beide Prinzipe nicht etwa selbständig, sondern setzen sich als Einheit, als Ineinander von Licht und Dunkel. Das in dieser Weise in sich selbst getrübte, verdunkelte Licht, das aber ebenso das Dunkle durchdringt und durchleuchtet, gibt das Prinzip für die Farbe als eigentliches Material der Malerei ... "20. Hegel beschreibt damit die Helldunkel-Farbe, die „luminaristische Farbe ${ }^{\text {‘c21 }}$, Rembrandts künstlerisches Material schlechthin.

Entspricht nun diesem Gestaltungsprinzip ein besonderer Inhalt? In Hegels „Ästhetik“ liest man: „Der wahre Inhalt des Romantischen ist die absolute Innerlichkeit, die entsprechende Form die geistige Subjektivität, als Erfassen ihrer Selbständigkeit und Freiheit. Dies in sich Unendliche und an und für sich Allgemeine ist die absolute Negativität von allem Besondern, die einfache Einheit mit sich, die alles Außereinander, alle Prozesse der Natur und deren Kreislauf des Entstehens, Vergehens und Wiedererstehens, alle Beschränktheit des geistigen Daseins verzehrt und alle besonderen Götter zu der reinen unendlichen Identität mit sich aufgelöst hat. In diesem Pantheon sind alle Götter entthront, die Flamme der Subjektivität hat sie zerstört, und statt der plastischen Vielgötterei kennt die Kunst jetzt nur einen Gott, einen Geist, eine absolute Selbständigkeit, welche als das absolute Wissen und Wollen ihrer selbst mit sich in freier Einheit bleibt und nicht mehr zu jenen besonderen Charakteren und Funktionen auseinanderfällt, deren einziger Zusammenhalt der Zwang einer dunkeln Notwendigkeit war ..."22.

Die romantische Kunstform ist die der christlichen Religion. In der neuzeitlichen Malerei aber durchdringen sich, anders als Hegel es darstellte, Helldunkel und Plastik, es durchdringen sich christliche und mythologische Gehalte, sie durchdringen sich und lassen sich gleichwohl auch voneinander trennen.

Wie aber verhalten sich dazu die unterschiedlichen Zeitgestalten?

20 Hegel, ebenda, Bd. 14, S. 15, 25, 25 f.

21 Vgl. Ernst Strauss, Koloritgeschichtliche Untersuchungen zur Malerei seit Giotto und andere Studien, hrsg. von Lorenz Dittmann, München, Berlin 1983, 17-26.

22 Hegel (wie Anm. 19) $122 \mathrm{f}$. 
Die mythische, die zyklische Zeit ist die geschlossene, die „plastische“ Zeitgestalt.

Die christliche, die eschatologische Zeit jedoch durchbricht letztlich jede bildnerische Geschlossenheit. Im Licht und seiner Spannung zum Dunkel, in der Sammlung des Lichts auf eine transzendierende Mitte, eine ,absolute“ Gegenwart, im Verweis des Lichts auf ein TranszendentZukünftiges, in Licht und Dunkel als anschaulichem Symbol von Eröffnung und Verbergung eines „Inneren“, kann sie künstlerische Gestalt gewinnen. 